\title{
An integral representation for the Riemann zeta function on positive integer arguments
}

\author{
Sumit Kumar Jha \\ IIIT-Hyderabad, India \\ Email: kumarjha.sumit@research.iiit.ac.in
}

May 23, 2019

\begin{abstract}
In this brief note, we give an integral representation for the Riemann zeta function for positive integer arguments. To the best of our knowledge, the representation is new.

Keywords: Ramanujan's Master Theorem; Riemann Zeta Function; Polygamma Function; Polylogarithm Function

AMS Classification: $33 \mathrm{E} 20$
\end{abstract}

We prove the following

Theorem 1. We have, for integers $r \geq 2$,

$$
\zeta(r)=\frac{1}{\pi\left(2-2^{r}\right)} \int_{0}^{\infty} \frac{x^{-1 / 2} \mathbf{L i}_{r}(-x)}{1+x} d x
$$

where $\mathbf{L i}_{r}(x)=\sum_{k=1}^{\infty} \frac{x^{k}}{k^{r}}$ is the polylogarithm function, and $\zeta(r)=\sum_{n=1}^{\infty} \frac{1}{n^{r}}$ is the Riemann zeta function.

The above can be obtained as a direct consequence of the following result

Theorem 2. For integers $r \geq 2$ and $0<n<1$, we have

$$
\int_{0}^{\infty} x^{n-1} \frac{\mathbf{L i}_{r}(-x)}{1+x} d x=\frac{\pi}{\sin n \pi}\left(\zeta(r)+(-1)^{r-1} \frac{\psi_{r-1}(1-n)}{(r-1) !}\right) .
$$

where $\mathbf{L i}_{r}(x)=\sum_{k=1}^{\infty} \frac{x^{k}}{k^{r}}$ is the polylogarithm function, $\psi_{n}(x)=\frac{d^{n}}{d x^{n}} \frac{\Gamma^{\prime}(x)}{\Gamma(x)}$ is the Polygamma function, $\Gamma(x)$ is the Gamma function, and $\zeta(r)$ is the Riemann zeta function.

Proof. Let

$$
H_{n}^{(r)}=\sum_{k=1}^{n} \frac{1}{k^{r}}
$$

be the generalized Harmonic number. Then, we have the following generating function from [2]

$$
\frac{\mathbf{L i}_{r}(x)}{1-x}=\sum_{n=1}^{\infty} H_{n}^{(r)} x^{n}
$$

for $|x|<1$. We can also write

$$
\frac{\mathbf{L i}_{r}(-x)}{(1+x)}=\sum_{n=1}^{\infty} H_{n}^{(r)}(-x)^{n} .
$$

We have for the following explicit form from [3]

$$
H_{n}^{(r)}=\zeta(r)+(-1)^{r-1} \frac{\psi_{r-1}(n+1)}{(r-1) !} .
$$


First note in this form, we have, $H_{0}^{(r)}=\zeta(r)+(-1)^{r-1} \frac{\psi_{r-1}(1)}{(r-1) !}=0$. Next, we use Ramanujan's Master Theorem (RMT) from [1], which is,

$$
\int_{0}^{\infty} x^{s-1}\left\{\phi(0)-x \phi(1)+x^{2} \phi(2)-\cdots\right\} d x=\frac{\pi}{\sin s \pi} \phi(-s),
$$

where the integral is convergent for $0<\boldsymbol{R e}(s)<1$, and after certain conditions are satisfied by $\phi$. Now, using RMT with equations (3) and (4) gives us required equation (2).

Proof of Theorem 1. We get the required equation (1) by plugging in $n=1 / 2$ in equation (2), and using the following fact from [3]

$$
\psi_{r-1}\left(\frac{1}{2}\right)=(-1)^{r} \cdot(r-1) ! \cdot\left(2^{r}-1\right) \cdot \zeta(r) .
$$

REFERENCES

1. Amdeberhan, T., Espinosa, O., Gonzalez, I., Harrison, M., Moll, V. H., \& Straub, A. (2012). Ramanujan's master theorem. The Ramanujan Journal, 29(1-3), 103-120.

2. Weisstein, Eric W. "Polylogarithm." From MathWorld-A Wolfram Web Resource.

3. Weisstein, Eric W. "Polygamma Function." From MathWorld-A Wolfram Web Resource 